\title{
Importância da validação dos modelos de risco cardiovascular nos cuidados de saúde primários
}

Maria Gouveia, ${ }^{1}$ Inês Pimenta Rodrigues, ${ }^{2}$ Daniel Pinto ${ }^{3-4}$

\section{RESUMO}

Introdução: As doenças cardiovasculares são a principal causa de morte nos países desenvolvidos e os eventos cardiovasculares nãofatais contribuem para morbilidade significativa na população destes países.

Modelos de risco cardiovascular usados atualmente: Os modelos de avaliação de risco cardiovascular, tendo em conta a conjugação de fatores de risco presentes num doente, atribuem um valor essencialmente quantitativo ao risco cardiovascular individual para determinados eventos fatais ou não fatais e permitem estabelecer metas para início de medidas terapêuticas preventivas. Entre os modelos de avaliação de risco cardiovascular mais amplamente usados estão o Systematic Coronary Risk Evaluation (SCORE) e as equações de Framingham.

Estudos de validação dos modelos de risco cardiovascular: Não obstante a sua utilidade clínica, estudos europeus concluíram que estes modelos sobrestimam o risco de eventos cardiovasculares fatais e não-fatais observados quando aplicados a populações diferentes daquela em que foram desenvolvidos.

Discussão: Atualmente não existe literatura que tenha validado a aplicação destes modelos de risco na população portuguesa, pelo que não podemos saber se estes modelos são suficientemente fiáveis na nossa população e se deverão ser usados para guiar decisões na prática clínica.

Palavras-chave: Doenças cardiovasculares; Modelos cardiovasculares; Estudos de validação; Cuidados de saúde primários.

\section{INTRODUÇÃO}

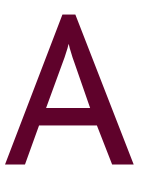

s doenças cardiovasculares são a principal causa de morte nos países desenvolvidos e os eventos cardiovasculares não-fatais contribuem para morbilidade significativa na população destes países. Segundo dados de 2012, a taxa de mortalidade padronizada relativa a isquémia cardíaca na União Europeia foi de 137 óbitos por 100.000 habitantes, estando Portugal abaixo da média com uma taxa de mortalidade padronizada por isquémia inferior a 100 óbitos por 100.000 habitantes. ${ }^{1}$ Dados de 2014 obtidos pelo Instituto Nacional de Estatística documentam que cerca de $40 \%$ dos óbitos na população com idades entre os 30 e 74 anos ocorreram por doenças do aparelho circulatório. ${ }^{2}$

1. Médica Interna de Medicina Geral e Familiar. USF São Julião, ACeS Lisboa Ocidental e Oeiras.

2. Médica Interna de Medicina Intensiva. Hospital Garcia de Orta, EPE.

3. Médico de família. USF São Julião, ACeS Lisboa Ocidental e Oeiras.

4. Unidade de Medicina Geral e Familiar, NOVA Medical School, Faculdade de Ciên-

cias Médicas, Universidade Nova de Lisboa.
Desta forma, a prevenção de eventos cardiovasculares fatais e não-fatais ocupa, atualmente, um lugar primordial na atividade dos cuidados de saúde primários. É prática comum e recomendada a identificação de fatores de risco para ocorrência de eventos cardiovasculares e modificação destes mesmos fatores com alteração de estilo de vida ou terapêutica farmacológica, de acordo com o risco cardiovascular individual calculado através de modelos matemáticos. ${ }^{3}$ Tendo em conta a conjugação e sinergismo entre alguns dos fatores de risco presentes, os modelos de risco atribuem um valor essencialmente quantitativo ao risco individual para determinados eventos cardiovasculares fatais ou não fatais, o que permite estabelecer metas para início de medidas terapêuticas preventivas custo-efetivas. ${ }^{3-4}$

\section{Modelos de risco cardiovascular usados atualmente}

Entre os modelos de avaliação de risco cardiovascular mais amplamente usados estão o Systematic Coronary Risk Evaluation (SCORE) para estimativa do risco de eventos 
cardiovasculares fatais a dez anos e os modelos de risco para diferentes outcomes desenvolvidos a partir das coortes dos Framingham Heart Studye Framingham Offspring Study.

Os modelos de avaliação de risco cardiovascular de Framingham foram desenvolvidos nos Estados Unidos da América (EUA) utilizando a amostra dos American Framingham Heart Study e Framingham Offspring Study. A partir desta amostra, selecionando os participantes com idades compreendidas entre os 30 e os 74 anos, sem história prévia de doença cardiovascular, foram desenvolvidos seis modelos de risco cardiovascular que diferem nos outcomes avaliados e na combinação de fatores de risco utilizada para obter um valor de risco numérico. ${ }^{5-6}$ Como modelo de risco cardiovascular mais abrangente existe a equação para estimativa do risco de evento cardiovascular fatal ou não-fatal a dez anos, desenvolvida a partir de 8.491 participantes com seguimento a doze anos. ${ }^{5}$ Esta equação expressa a probabilidade individual do doente, num período de dez anos, experienciar um dos seguintes eventos: enfarte agudo do miocárdio (EAM), angina, insuficiência coronária, acidente vascular cerebral (AVC) isquémico ou hemorrágico, acidente isquémico transitório (AIT), doença arterial periférica e insuficiência cardíaca ou morte por causa cardiovascular. Apesar do peso dos vários fatores de risco influenciar cada um destes eventos de forma diferente, é útil na prática clínica diária, especialmente no contexto de prevenção primária, a existência de um calculador de risco abrangente e facilmente aplicável para determinar a probabilidade de doença aterosclerótica nos diferentes territórios vasculares. Ao longo dos anos foram desenvolvidos vários modelos de risco cardiovascular, tendo por base a amostra do Framingham Heart Study, sendo as variáveis incluídas no modelo de risco cardiovascular global as seguintes: idade, sexo, tabagismo ativo, diabetes, pressão arterial, colesterol total, colesterol HDL e tratamento farmacológico para hipertensão arterial. Quando o risco cardiovascular global de Framingham é igual ou superior a $20 \%$, estes são os indivíduos considerados de alto risco a quem devem ser instituídas medidas terapêuticas preventivas..$^{5-6}$

Outro dos resultados mais utilizados nas equações de Framinghamé a estimativa de risco de eventos coronários, uma vez que estes continuam a ser uma grande causa de mortalidade e morbilidade em muitos países desenvolvidos. Neste caso, as variáveis utilizadas para cálculo de risco são a idade, sexo, tabagismo ativo, diabetes, categoria de hipertensão segundo o Fifth Joint National Committee on Hypertension (JNC-V), categoria de colesterol total ou LDL segundo o National Cholesterol Education Program, Adult Treatment Panel II (NCEP ATP II) e colesterol HDL. ${ }^{7-8}$

O modelo SCORE é um sistema de estimativa de risco cardiovascular desenvolvido em países europeus pela constatação de que o modelo Framingham, desenvolvido nos EUA, muitas vezes não se aplicava à população europeia, sobrestimando o risco. Houve, assim, a necessidade de desenvolver um modelo aplicável à população europeia para utilização na prática clínica. ${ }^{9} \mathrm{O}$ modelo SCORE engloba duas equações de risco cardiovascular que diferem ao serem aplicáveis a regiões consideradas de alto risco ou a regiões de baixo risco cardiovascular. ${ }^{10}$ A equação para regiões de alto risco cardiovascular foi desenvolvida utilizando estudos de coorte da Dinamarca, Finlândia e Noruega, países onde se registam mais eventos cardiovasculares tendo em conta ponderação para fatores de risco e outras interferências. Estudos de coorte de Itália, Bélgica e Espanha foram utilizados para desenvolver equações para regiões de baixo risco, nas quais Portugal se insere. Ambos os modelos SCORE avaliam o risco de morte cardiovascular em doentes entre os 40 e 65 anos considerando as seguintes variáveis: idade, sexo, tabagismo ativo, colesterol total e HDL e pressão arterial sistólica. As metas para instituição de tratamento incluem $3 \%$ e $5 \%$ para regiões de baixo risco e $3 \%, 5 \%, 7 \%$ e $10 \%$ para as regiões de alto risco. ${ }^{10}$

De notar que, no projeto SCORE, diferentemente do Framingham, foram apenas incluídos os eventos fatais como forma de diminuir a subjetividade do modelo, uma vez que a definição de doença cardiovascular não-fatal cria problemas tanto na criação de um modelo de risco como na aplicabilidade e replicação do modelo a populações diferentes daquela nas quais foi desenvolvido. Outra característica que difere entre estes dois modelos é a não inclusão da diabetes como uma variável para o cálculo de risco no SCORE. Isto deve-se ao facto de a informação acerca do diagnóstico de diabetes não ter sido consistentemente obtida nos estudos de coorte que serviram de base ao desenvolvimento do SCORE e, portanto, os autores consideraram não ter informação suficiente para incluir este critério na quantificação do risco. ${ }^{10}$

É importante lembrar que, não obstante a sua utilidade clínica, qualquer modelo de risco consiste numa simplificação da totalidade de fatores de risco presentes num indivíduo. No caso dos modelos acima considerados não são avaliados parâmetros como raça, etnia, índice de massa corporal (IMC), alimentação, prática de exercício físico, história familiar de doença cardiovascular, novos marcadores de risco cardiovascular e outros fatores genéticos ou 
ambientais. Tudo isto pode levar a erros na previsão da probabilidade de eventos cardiovasculares em diferentes populações. ${ }^{11}$

Em Portugal, a Norma de Orientação Clínica da Direção-Geral da Saúde n. ${ }^{\circ}$ 005/2013 determina a avaliação do risco cardiovascular através do modelo SCORE a utentes com idade entre os 40 e 65 anos. Segundo esta norma, os utentes são classificados em quatro grupos de risco distintos (risco muito alto, alto, moderado ou baixo). Há situações que dispensam a avaliação pelo SCORE e os utentes são logo classificados como tendo risco muito alto (presença de doença cardiovascular aterosclerótica documentada; diabetes mellitus (DM) tipo 1 ou 2, com um ou mais fatores de risco cardiovascular e/ou lesão dos órgãosalvo; doença renal crónica grave) ou risco alto (fatores de risco vascular isolados muito elevados; DM tipo 1 ou 2, mas sem outros fatores de risco cardiovascular ou lesão de órgão-alvo; doença renal crónica moderada). A terapêutica é recomendada naqueles com risco alto ou muito alto, que inclui doentes com avaliação SCORE igual ou superior a $5 \%$ e tem o objetivo de reduzir o risco de eventos cardiovasculares. $^{3}$

\section{Estudos de validação dos modelos de risco cardiovascular}

Estudos anteriores, de uma forma geral, concluíram que os modelos de risco cardiovascular sobrestimam o risco de eventos fatais e não-fatais efetivamente observados na população. Um estudo realizado no Reino Unido concluiu que as equações de risco do estudo de Framingham sobrestimam em $57 \%$ o risco de eventos coronários fatais ou não-fatais de forma constante em todos os níveis de ris$\mathrm{co}$, tanto que quanto maior o risco efetivo maior o risco absoluto calculado correspondente. ${ }^{4}$ Este mesmo estudo sugere ainda que existe uma desadequação dos valores sugeridos como meta para início de tratamento, argumentando que o valor de $30 \%$, sugerido para o Reino Unido, apresenta baixas sensibilidade e especificidade na identificação de utentes que beneficiam de tratamento. Os autores propõem valores bastante inferiores como meta, sugerindo como marcador para início de tratamento um risco de $15 \%$ de forma a aumentar a sensibilidade, levando, contudo, a uma redução da especificidade. Por outro lado, os autores notam ser possível uma recalibração do modelo de risco de Framingham utilizado, uma vez que o risco é sobrestimado de forma constante em todos os níveis, sendo que, após recalibração, o risco previsto pelo modelo se aproxima do risco real da amostra. ${ }^{4}$ Da mesma for- ma, outros estudos europeus concluíram que tanto as equações de Framingham como o modelo SCORE sistematicamente sobrestimam o risco real em até $50 \%$. Estes resultados parecem demonstrar diferenças entre as populações, em qual o modelo de risco foi desenvolvido e aquela a que é aplicado, o que questiona a validade do uso destes modelos na Europa. ${ }^{12-13}$

Por outro lado, num estudo realizado com uma amostra de mulheres australianas, apesar de ter demonstrado que tanto o risco de morte cardiovascular estimado pelo Framingham como pelo modelo SCORE para regiões de baixo risco sobrestimam o risco real da população, a percentagem relativa de sobrestimação do risco foi de apenas $15 \%$, muito inferior a estudos europeus. Neste estudo, estes modelos de risco foram considerados calibrados e, portanto, adequados para prever o risco de mortalidade cardiovascular nesta população. Já os limiares de tratamento propostos foram considerados como inadequados para identificação de utentes que beneficiam de tratamento dirigido a fatores de risco e os autores propõem tratamento a partir de estimativas de risco mais baixas, de modo a melhorar a identificação destes utentes. ${ }^{11}$

Como exceção, existe ainda a população de diabéticos tipo 2, na qual os modelos de risco de Framingham parecem subestimar a taxa de eventos observada e o SCORE, já recalculado consoante a sugestão dos autores para a população diabética, parece sobrestimar este risco. ${ }^{14}$ No entanto, nenhum destes modelos de risco está validado na população de diabéticos. Apesar de equações do modelo de Framingham incorporarem a diabetes como uma variável, o número de diabéticos incluídos na amostra foi reduzido e a diabetes foi considerada de forma categórica, ou seja, sem atenção ao controlo glicémico ou gravidade da doença. ${ }^{14}$

Outra limitação do uso destes modelos de avaliação de risco cardiovascular é o facto de terem sido desenvolvidos e, portanto, validados apenas em pessoas até aos 65 anos no caso do SCORE e 74 anos para as equações de Framingham. Um estudo de 2009, realizado na Holanda, confirmou a não aplicabilidade do risco pelas equações de Framingham à população com mais de 85 anos sem história prévia de doença cardiovascular. São resultados em concordância com outros estudos que, da mesma forma, demonstram uma maior desadequação das equações de risco nos extremos de idade, provavelmente por alteração da contribuição relativa dos diferentes fatores de risco na produção de eventos cardiovasculares. ${ }^{15-16}$ No entanto, a prevalência e incidência de eventos cardiovasculares fatais 
e não fatais aumenta exponencialmente com a idade e, tendo em conta o envelhecimento da população e o aumento da esperança média de vida, que se tem vindo a verificar nas últimas décadas, a maioria dos eventos cardiovasculares ocorre em faixas etárias nas quais os modelos de risco não são aplicáveis mas que, em teoria, poderiam ainda ser alvo da aplicação de medidas de prevenção primária. Alguns autores sugerem a utilização de medidas preventivas na população idosa com o argumento de que a intervenção nestas faixas etárias, apesar de reduzir de forma modesta o risco relativo, tem um grande impacto sobre o risco cardiovascular absoluto. ${ }^{17-19}$

Assim, apesar do modelo Framingham estar validado em populações dos EUA, Austrália e Nova Zelândia, o seu uso a nível europeu muitas vezes sobrestima o risco e deve ser sujeito a ajustes e calibração adicional para ser utilizado.9,11 Para o modelo SCORE, não obstante ter sido desenvolvido em diversos países europeus, foram igualmente relatadas incoerências entre o risco calculado através deste modelo e o risco observado numa determinada população. ${ }^{13-14}$

\section{DISCUSSÃO}

A tendência da medicina atual é utilizar, cada vez mais, modelos para estimativa de risco e estabelecimento de metas como forma de auxiliar a decisão terapêutica. As principais vantagens destes modelos são o facto de facilitarem a prática clínica ao relacionar os fatores de risco mais importantes presentes num utente, permitindo uma avaliação rápida numa altura em que os médicos são cada vez mais pressionados a cumprir um maior número de consultas num curto período de tempo. Por outro lado, uma vez que estes modelos categorizam os utentes de acordo com o seu grau de risco, podem ser uma ferramenta útil em termos de educação para a saúde.

Como referido previamente, em Portugal, a Norma de Orientação Clínica da Direção-Geral da Saúde n. ${ }^{\circ}$ 005/2013 determina a avaliação do risco cardiovascular através do modelo SCORE a utentes com idade entre os 40 e 65 anos. Segundo esta norma, os utentes são classificados em quatro grupos de risco distintos (risco muito alto, alto, moderado ou baixo) que determinam a utilização de medidas mais intensivas de prevenção, incluindo medidas farmacológicas. $^{3}$

Não obstante as vantagens dos modelos de risco cardiovascular, até hoje estes modelos não foram validados na população portuguesa, pelo que não podemos saber a sua fiabilidade na nossa população. De acordo com estudos realizados noutros países europeus, frequentemente sobrestimam o risco cardiovascular. Outro aspeto a ter em conta é a população na qual estes modelos foram desenvolvidos e à qual devem ser aplicados. Por exemplo, a ferramenta para cálculo do risco cardiovascular do SClínico, sistema de registo informático utilizado por diversas unidades de cuidados de saúde primários, permite o cálculo do risco cardiovascular fora das idades que foram validadas pelo SCORE, o que constitui igualmente um problema de fiabilidade na utilização desta ferramenta.

Assim sendo, será razoável incentivar a utilização sistemática de modelos de risco que não estão validados na população portuguesa? Sabendo ainda que as medidas preventivas incluem medidas farmacológicas, e que estas não são isentas de efeitos secundários, será que devemos aplicar estes modelos de uma forma rígida para guiar decisões como a prescrição de um novo fármaco?

Atualmente, perante a grande variedade de modelos de risco que foram desenvolvidos em diferentes populações, o principal problema com a sua utilização é a ausência de dados acerca da sua aplicação na prática clínica, particularmente em populações diferentes daquelas em que foram desenvolvidos; o que torna os estudos de validação um importante passo no futuro da investigação científica desta área, mais do que o desenvolvimento de novos modelos com integração de diferentes fatores de risco. ${ }^{20}$

Um estudo de validação dos modelos de risco cardiovascular permitiria compreender se existe concordância entre o risco estimado pelos modelos e o risco observado na população portuguesa e determinar a necessidade de ajustes ou calibração dos mesmos. De uma forma geral, a validação externa seria importante para tornar estes modelos mais fiáveis para aplicação à população portuguesa. Até lá, os profissionais de saúde devem encarar estes modelos como estimativas aproximadas do risco, com margens de erro importantes e tendência a que o risco estimado seja superior ao real. Assim, a decisão de iniciar ou não uma determinada intervenção preventiva deve ter em conta uma avaliação global de todos os fatores de risco, incluindo aqueles que não fazem parte destes modelos.

\section{REFERÊNCIAS BIBLIOGRÁFICAS}

1. EUROSTAT. Estatísticas sobre causas de morte [Internet]. Luxembourg: EUROSTAT; 2016 [cited 2016 Apr 10; updated 2017 May]. Available from: http://ec.europa.eu/eurostat/statistics-explained/index.php/Causes_of_ death_statistics/pt\#An.C3.A1lise_por_idade

2. Instituto Nacional de Estatística. Óbitos $\left(n .^{\circ}\right)$ por local de residência (NUTS - 2013) [Internet]. Lisboa: INE; 2016 [cited 2016 Apr 10; updated 2018 Mar 16]. Available from: https://www.ine.pt/xportal/xmain?xpid=INE\&xpgid $=$ ine_indicadores\&indOcorrCod $=0008206 \&$ contexto $=b d \&$ selTab $=$ tab2 
3. Direção-Geral da Saúde. Avaliação do risco cardiovascular SCORE: norma n. ${ }^{\circ}$ 005/2013, de 19/03/2013, atualizada em 21/01/2015. Lisboa: DGS; 2015.

4. Brindle P, Emberson J, Lampe F, Walker M, Whincup P, Fahey T, et al. Predictive accuracy of the Framingham coronary risk score in British men: prospective cohort study. BMJ. 2003;327(7426):1267.

5. D'Agostino RB Sr, Vasan RS, Pencina MJ, Wolf PA, Cobain M, Massaro JM, et al. General cardiovascular risk profile for use in primary care: the Framingham Heart Study. Circulation. 2008;117(6):743-53.

6. Anderson KM, Odell PM, Wilson PW, Kannel WB. Cardiovascular disease risk profiles. Am Heart J. 1991;121(1 Pt 2):293-8.

7. Wilson PW, D'Agostino RB, Levy D, Belanger AM, Silbershatz H, Kannel WB. Prediction of coronary heart disease using risk factor categories. Circulation. 1998;97(18):1837-47.

8. Framingham Heart Study. Coronary heart disease: 10-year risk [Internet]. Framingham Heart Study; 2016 [cited 2016 Aug 16]. Available from: https://www.framinghamheartstudy.org/fhs-risk-functions/coronary-heartdisease-10-year-risk/

9. Eichler K, Puhan MA, Steurer J, Bachmann LM. Prediction of first coronary events with the Framingham score: a systematic review. Am Heart J. 2007;153(5):722-31,731.e1-8.

10. Conroy RM, Pyörälä K, Fitzgerald AP, Sans S, Menotti A, De Backer G, et al. Estimation of ten-year risk of fatal cardiovascular disease in Europe: the SCORE project. Eur Heart J. 2003;24(11):987-1003.

11. Goh LG, Welborn TA, Dhaliwal SS. Independent external validation of cardiovascular disease mortality in women utilising Framingham and SCORE risk models: a mortality follow-up study. BMCWomens Health. 2014; 14:118.

12. Menotti A, Puddu PE, Lanti M. Comparison of the Framingham risk function-based coronary chart with risk function from an Italian population study. Eur Heart J. 2000;21(5):365-70.

13. Ulmer H, Kollerits B, Kelleher C, Diem G, Concin H. Predictive accuracy of the SCORE risk function for cardiovascular disease in clinical practice: a prospective evaluation of 44,649 Austrian men and women. Eur J Cardiovasc Prev Rehabil. 2005;12(5):433-41.

14. Coleman RL, Stevens RJ, Retnakaran R, Holman RR. Framingham, SCORE, and DECODE risk equations do not provide reliable cardiovascular risk estimates in type 2 diabetes. Diabetes Care. 2007;30(5):1292-3.

15. de Ruijter W, Westendorp RG, Assendelft WJ, den Elzen WP, de Craen AJ, le Cessie $\mathrm{S}$, et al. Use of Framingham risk score and new biomarkers to predict cardiovascular mortality in older people: population based observational cohort study. BMJ. 2009;338:a3083.

16. Kannel WB. Coronary heart disease risk factors in the elderly. Am J Geriatr Cardiol. 2002;11(2):101-7.

17. Dornbrook-Lavender KA, Pieper JA, Roth MT. Primary prevention of coronary heart disease in the elderly. Ann Pharmacother. 2003;37(11):1654-63.

18. Andrawes WF, Bussy C, Belmin J. Prevention of cardiovascular events in elderly people. Drugs Aging. 2005;22(10):859-76

19. Kannel WB, D'Agostino RB. The importance of cardiovascular risk factors in the elderly. Am J Geriatr Cardiol. 1995;4(2):10-23.

20. Damen JA, Hooft L, Schuit E, Debray TP, Collins GS, Tzoulaki I, et al. Prediction models for cardiovascular disease risk in the general population: systematic review. BMJ. 2016;353:i2416.

\section{CONFLITO DE INTERESSES}

Os autores declaram não possuir quaisquer conflitos de interesse.

\section{ENDEREÇO PARA CORRESPONDÊNCIA}

Maria Gouveia

E-mail: maria.folque.gouveia@gmail.com

http://orcid.org/0000-0003-0796-813X

Recebido em 02-02-2017

Aceite para publicação em 23-03-2018

\section{ABSTRACT}

\section{VALIDATION'S IMPORTANCE OF CARDIOVASCULAR RISK CALCULATION IN PRIMARY HEALTH CARE}

Introduction: Cardiovascular diseases are the most important cause of death in developed countries and non-fatal cardiovascular events contribute to significant morbidity in the population of these countries.

Cardiovascular risk models currently used: Cardiovascular risk models consider the sum of risk factors present in a patient, attribute a quantitative value to the cardiovascular risk to certain fatal and non-fatal events and allow us to establish cut-offs to introduce preventive therapeutic measures. Among the most used cardiovascular risk models are the Systematic Coronary Risk Evaluation (SCO$\mathrm{RE})$ and Framingham equations.

Validation studies of cardiovascular risk models: Despite their clinical utility, European studies showed that these models overestimate the observed risk of fatal and non-fatal cardiovascular events when applied to populations different from those they were derived from.

Discussion: Currently there is no literature validating the use of these cardiovascular risk models in the Portuguese population, hence there is uncertainty whether or not the recommended models accurately measure risk in our population and if they should be used in clinical practice.

Keywords: Cardiovascular diseases; Cardiovascular models; Validation studies; Primary health care 